\title{
Análisis de la corrosión de uniones por soldadura fuerte de compósitos matriz cobre
}

\author{
Analysis of corrosion of joints brazing \\ copper matrix composites
}

\author{
David Cabrera-de la Cruz ${ }^{1}$, Carlos Alberto León Patiño ${ }^{1}$, \\ Ricardo Galván-Martínez ${ }^{2}$
}

\author{
${ }^{1}$ Instituto de Investigación en Metalurgia y Materiales, Universidad Michoacana de San Nicolás de Hidalgo Apdo. Postal \\ 888 Centro, C.P. 58000, Morelia, Michoacán, México. \\ e-mail: cabreraccd@gmail.com; caleon@umich.mx \\ ${ }^{2}$ Unidad Anticorrosión, Instituto de Ingeniería, Universidad Veracruzana Av. S.S. Juan Pablo II. S/N, Zona Universitaria, \\ Fracc. Costa Verde. CP. 94294, Veracruz, Veracruz, México. \\ e-mail: rigalvan@uv.mx
}

\section{RESUMEN}

En el presente trabajo de investigación se estudió el proceso electroquímico de la corrosión en agua de mar sintética de la unión disímil por soldadura fuerte del compósito TiC/Cu. Los compósitos fueron fabricados mediante infiltración líquida sin el uso de presión externa. Dichos compuestos se unieron a cobre usando una aleación de $\mathrm{Cu}-\mathrm{Ag}$ con adición de Ti en polvo. Estas juntas mostraron una excelente adhesión, formando un ensamble disímil pero coherente. Las pruebas de corrosión empleadas fueron las técnicas electroquímicas de curvas de polarización (CP) y ruido electroquímico (RE). Para determinar el tiempo de estabilización de las reacciones anódica y catódica del proceso de corrosión, se realizó un seguimiento de potencial de corrosión $\left(\mathrm{E}_{\text {corr }}\right)$ con respecto al tiempo. Las mediciones se realizaron a diferentes intervalos de tiempo, durante un tiempo total de exposición de 24 horas. Las mediciones electroquímicas se realizaron en una celda electroquímica con un arreglo típico de tres electrodos, un electrodo de trabajo, un electrodo de referencia (calomel) y un electrodo auxiliar (barra de grafito). Los resultados electroquímicos muestran que la unión (TiC/Cu-Cu) presenta mayor susceptibilidad a corrosión localizada comparada con el metal base. El mecanismo de corrosión de las uniones se generó por una celda de aeración diferencial producida por corrosión en hendidura y galvánica. Los resultados muestran una buena correlación entre el análisis superficial y los obtenidos por RE y CP.

Palabras clave: Corrosión, Compósito, Ruido Electroquímico, Soldadura fuerte.

\section{ABSTRACT}

This work presents an electrochemical corrosion study in synthetic seawater of the dissimilar joint of $\mathrm{TiC} / \mathrm{Cu}$ composite by brazing technique. Composites were machined by liquid infiltration without external pressure. This composite were joined with copper using a filler material constituted by an alloy of silver - copper and titanium powder. The composite joined shown a good adhesion forming a dissimilar assembled and coherent. In order to carry out the corrosion studies, two different techniques were used, potentiodynamic polarization curves (PCs) and electrochemical noise (EN). In addition, the corrosion potential measures as a function of the time were carried out to get the stabilization time of the anodic and cathodic reactions in the corrosion process. Electrochemical measurements were made at different exposure times with a total exposure time of 24 hours. A typical three-electrode electrochemical cell was used; where the working electrode (WE) was a sample of composite joined, the reference electrode was the saturated calomel electrode (SCE) and a sintered graphite rod was used as auxiliary electrode. The electrochemical results shown that the susceptibility to localized corrosion of the composite joined sample was higher than the based metal (copper). The corrosion mechanism of the composite joined sample is a differential aeration cell, where it can produce pitting and crevice corrosion form. The results shown a good correlation between the superficial analysis and the results 
obtained by the EN and CPs.

Keywords: Corrosion, Composites, Electrochemical Noise, Brazing.

\section{INTRODUCCIÓN}

$\mathrm{El} \mathrm{TiC} \mathrm{es} \mathrm{un} \mathrm{material} \mathrm{de} \mathrm{gran} \mathrm{importancia} \mathrm{para} \mathrm{la} \mathrm{industria} \mathrm{debido} \mathrm{a} \mathrm{sus} \mathrm{propiedades} \mathrm{físicas} \mathrm{y} \mathrm{químicas,} \mathrm{tales}$ como su alta dureza, punto de fusión, conductividad eléctrica y bajo coeficiente de expansión térmica utilizado para fabricar materiales compuestos [1,2], tal es el caso del compósito TiC/Cu.

La resistencia del material compuesto depende de la distribución homogénea del refuerzo, ya sea continuo o discontinuo [3]. En los materiales compuestos la resistencia al desgaste está influenciada por la cantidad de refuerzo y del tamaño de partícula. KARANTZALIS et al. [4] en sus estudios de compósitos de TiC con matriz de Ni-Al la dureza aumenta y el desgaste disminuye al aumentar la fracción en volumen de las partículas de TiC, como los observados por AKHTAR et al. [5] en materiales compuestos de $\mathrm{Cu}-\mathrm{Ti}-\mathrm{Al}$ y $\mathrm{Cu}$ $\mathrm{Ni}-\mathrm{Co}$, con distintos porcentajes de TiC. El proceso de soldadura fuerte constituye una alternativa interesante para uniones compósito-compósito y compósito-metal, al poner en contacto sus superficies y someterlas a una elevada temperatura aplicándoles una presión interfacial durante un intervalo de tiempo finito [6].

El método de soldadura fuerte se puede realizar enfrentando directamente las superficies a unir o utilizando un intermediario, FAN et al. [7] mencionan que el uso de la Ag-Cu-Ti y Ag-Cu-Zn [8] están siendo utilizados en uniones de materiales compuestos. El proceso de soldadura puede provocar la pérdida de esta distribución homogénea y crear zonas libres de refuerzo, grietas que posiblemente pueden conducir a fallas mecánicas tempranas dentro de las juntas de soldadura [9]. El comportamiento de la corrosión de los materiales compuestos de matriz metálica se decide por numerosos factores tales como la composición de la aleación, tamaño y distribución en el refuerzo y la naturaleza de la interfaz matriz-refuerzo, en sus uniones por soldadura pueden reducir la resistencia a la corrosión debido a la corrosión galvánica [10,11].

El estudio de diferentes técnicas electroquímicas en los fenómenos de corrosión proporciona información necesaria para predecir la velocidad de corrosión y su morfología en tiempos relativamente cortos. Como es el caso de la técnica electroquímica de Ruido Electroquímico (RE), es una técnica de corrosión no intrusiva que puede proporcionar información sobre los procesos sin alterar las reacciones, en la cual se pueden llevar a cabo mediciones de fluctuaciones de corriente y potencial, los cuales se pueden identificar por medio de un pulso de carga eléctrica constante a través del tiempo [12], y la técnica de curvas de polarización (CP), a diferencia de la técnica de RE esta es una técnica destructiva que acelera el proceso de corrosión, obteniendo información electroquímica como el potencial de corrosión $\left(\mathrm{E}_{\text {corr }}\right)$, la densidad de corriente $\left(\mathrm{i}_{\text {corr }}\right)$ y velocidad de corrosión $\left(\mathrm{V}_{\text {corr }}\right)$ [13].

El presente trabajo investiga la resistencia a la corrosión mediante la aplicación de las técnicas electroquímicas de Ruido Electroquímico (RE) y Curvas de Polarización (CP) de la unión de cobre metálico al compósito $\mathrm{TiC} / \mathrm{Cu}$ con un contenido cerámico del $60 \%$, utilizando una laminilla de aleación $\mathrm{Ag}-\mathrm{Cu}$ como intermediario. La microestructura del compósito y la unión disímil se caracterizaron por microscopía electrónica de barrido (SEM).

\section{MATERIALES Y MÉTODOS}

\subsection{Síntesis y unión disímil del compósito (TiC/Cu-Cu)}

Se utilizó la técnica de infiltración líquida sin el uso de presión externa para la obtención del compósito $\mathrm{TiC} / \mathrm{Cu}$. Se sinterizaron barras de $\mathrm{TiC}$ de 25 hasta $1250^{\circ} \mathrm{C}$, posteriormente fueron infiltrados con cobre a $1350^{\circ} \mathrm{C}$ durante 30 minutos. 


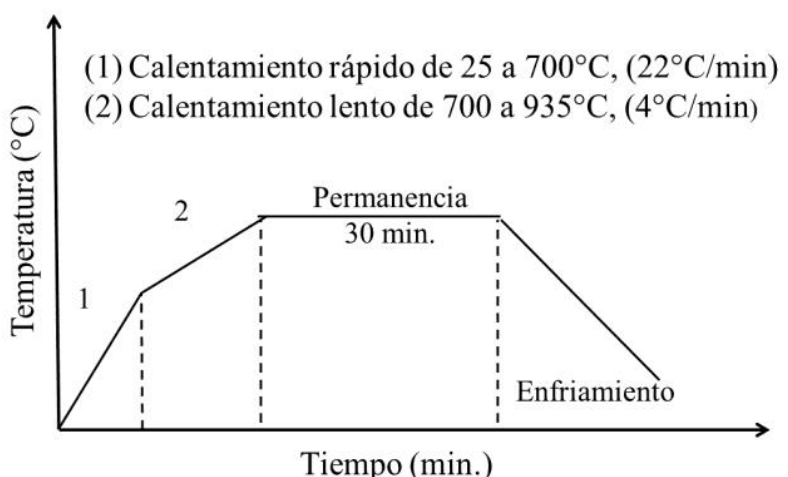

Figura 1: Ciclo de calentamiento del proceso de unión del compósito a cobre a $935^{\circ} \mathrm{C}$, bajo una atmosfera de argón.

Después de la obtención del compósito, se prepararon probetas de $1 \mathrm{x} 1 \mathrm{~cm}^{2}$ del compósito de TiC/Cu y de la aleación Ag-Cu (68-32 \% peso) con un espesor en el rango de 45-50 $\mu \mathrm{m}$, se adiciono Ti en polvo (Ti 99.7\%, malla -100, Aldrich) como un elemento activador del proceso de unión interfacial. La temperatura de trabajo se realizó en dos etapas como se presenta en la Figura 1, el tiempo de permanencia a $935{ }^{\circ} \mathrm{C}$ fue de 30 min. El proceso de sinterización, infiltración y unión se controló bajo una atmósfera dinámica de argón.

\subsection{Caracterización electroquímica}

Los electrodos de trabajo $(\mathrm{TiC} / \mathrm{Cu}-\mathrm{Cu})$ presentaron un área de exposición de $1 \mathrm{~cm}^{2}$ para las mediciones electroquímicas, como medio corrosivo se utilizó agua de mar sintética de acuerdo a la norma ASTM D-1141. Antes de realizar la experimentación, se sometieron a una limpieza mecánica con papel lija en una secuencia de 320, 400, 600, 800 y 1200. Posteriormente fueron enjuagadas con agua destilada y desengrasadas con acetona.

Para las mediciones electroquímicas de CP, la polarización fue de $\pm 500 \mathrm{mV}$ vs. $\mathrm{E}_{\text {corr }}$, utilizando una celda electroquímica, que contenía una barra de grafito como electrodo auxiliar, un electrodo de calomel saturado $\left(\mathrm{Hg} / \mathrm{Hg}_{2} \mathrm{Cl}_{2}\right)$ como electrodo de referencia y un electrodo de trabajo. Para la aplicación de la técnica de RE se sustituyó el grafito por otro electrodo de trabajo, midiéndose 1200 puntos, un punto por segundo.

\section{RESULTADOS}

\subsection{Compósito $\mathrm{TiC} / \mathrm{Cu}(60-40 \%$ vol.)}

Se obtuvo el compósito de carburo de titanio matriz cobre mediante la técnica de infiltración líquida sin el uso de presión externa utilizando atmósfera de argón de alta pureza. La Figura 2 muestra la microestructura del compósito $\mathrm{TiC} / \mathrm{Cu}$, así como el análisis de distribución atómica o mapeo en el compósito, la imagen muestra una distribución homogénea del refuerzo en la matriz.
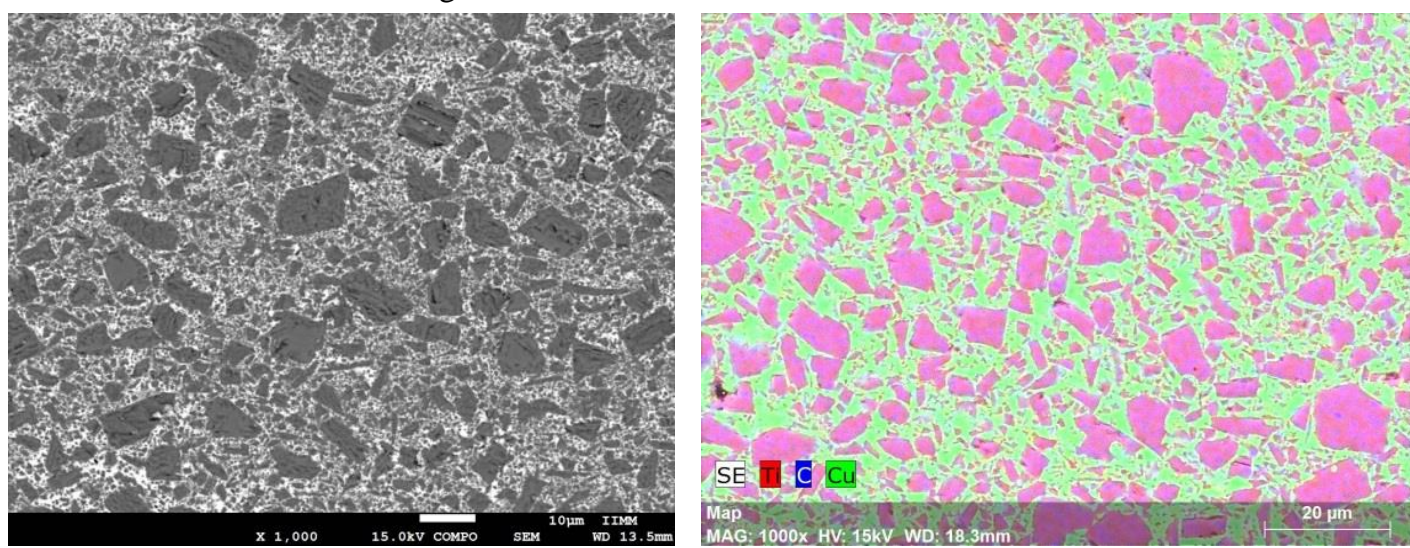

Figura 2: Micrografía y Mapeo del compósito TiC/Cu fabricado por infiltración capilar bajo una atmosfera de argón. 


\subsection{Unión brazing del compósito a cobre ( $\mathrm{TiC} / \mathrm{Cu}-\mathrm{Cu})$}

Los resultados del proceso de unión brazing del arreglo compósito - metal ( $\mathrm{TiC} / \mathrm{Cu}-\mathrm{Cu}$ ), se empleó la aleación de aporte de composición Ag-Cu (68-32 \%peso) más la adición de titanio que permite que la aleación humecte la superficie del compósito. Mediante la adición del elemento activo titanio, se logró la unión satisfactoria del compósito $\mathrm{TiC} / \mathrm{Cu}$ a probetas de cobre a partir de $935^{\circ} \mathrm{C}$ con un tiempo de permanencia de 30 minutos, la Figura 3 muestra la unión disímil pero coherente, la aleación de aporte funde y se une con la probeta de cobre, mostrando difusión de Ag en el cobre y compósito, lo que origina un excelente grado de adhesión de las contrapartes.

YU TANG et al. [14] mencionan que la adición de Ti influye en las reacciones interfaciales y desempeña un papel importante en la mojabilidad y la conectividad entre el metal o aleación y el cerámico.

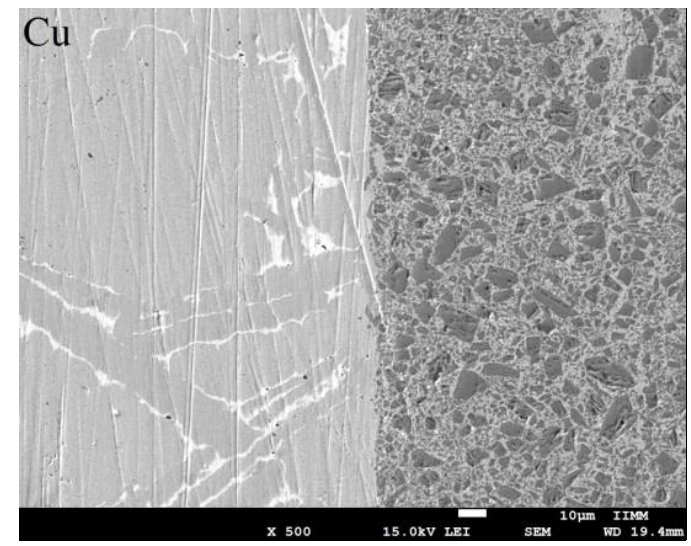

Figura 3: Micrografia de la unión disimil $\mathrm{TiC} / \mathrm{Cu} \bullet \mathrm{Ag}-\mathrm{Cu}+\mathrm{Ti} \bullet \mathrm{Cu}\left(935^{\circ} \mathrm{C}, 30 \mathrm{~min}\right)$.

La Figura 4 muestra el mapeo efectuado a la unión, donde se observa la buena distribución del $\mathrm{Cu}$ y TiC en el compósito, la aleación de aporte funde, mostrando difusión de la Ag en el cobre y compósito, lo que origina un excelente grado de adhesión de las contrapartes.
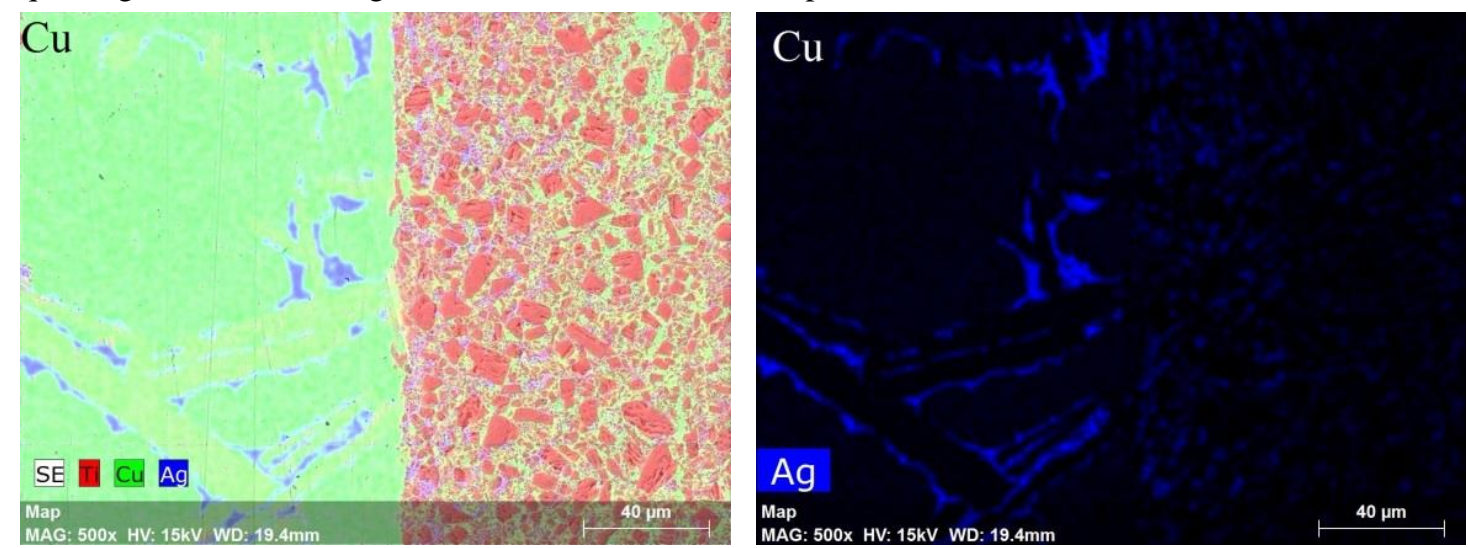

Figura 4: Mapeo de la difusión de $\mathrm{Ag}$ en la unión $\mathrm{TiC} / \mathrm{Cu} \cdot \mathrm{Ag}-\mathrm{Cu}+\mathrm{Ti} \bullet \mathrm{Cu}\left(935^{\circ} \mathrm{C}, 30 \mathrm{~min}\right)$.

\subsection{Estudio de la corrosión de la unión compósito-metal mediante RE}

La Figura 5 muestra las mediciones de potencial (E) y corriente (i) del ruido con respecto al tiempo, de la unión disímil TiC/Cu-Cu. Es importante mencionar que T0, T12 y T24 corresponden a los tiempos de exposición 0,12 y 24 horas respectivamente, de la unión inmersa en agua de mar sintética. 

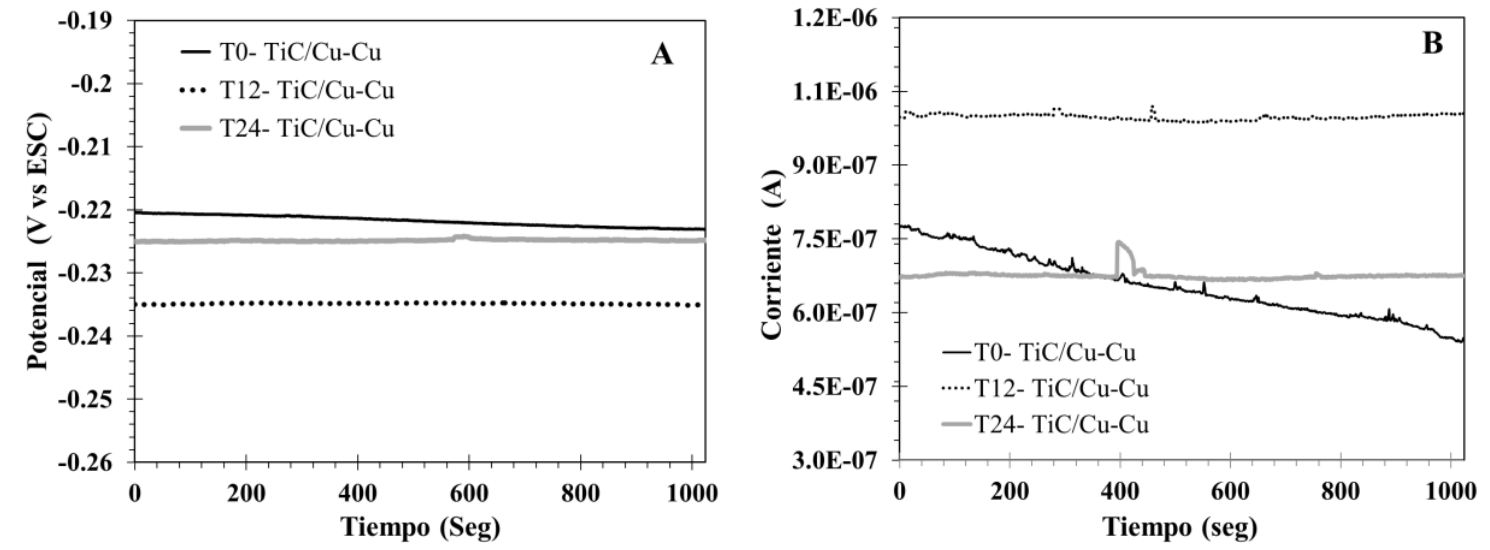

Figura 5: Mediciones de RE en las series de tiempo de la unión compósito-Cu en agua de mar sintética. Potencial vs tiempo (A), corriente vs tiempo (B).

En la Figura 5A se puede observar que el $\mathrm{E}_{\text {corr }}$ de la unión disímil al inicio y final de la prueba fueron más electropositivos que los valores obtenidos a las 12 horas de exposición, esta variación de potencial es atribui-do al proceso corrosivo generado por corrosión localizada como se observa en el la Figura 5B las oscilaciones estocásticas de corriente son indicativo de corrosión localizada, nucleación de picaduras y un proceso de corrosión en hendiduras, las fluctuaciones de corriente tienen un tiempo de vida muy corto, lo cual se atribu-ye a que existe una disolución del cobre en un solo punto.

\subsubsection{Resistencia del RE $\left(\mathbf{R}_{\mathbf{n}}\right)$}

La figura 6 muestra la comparación de los valores de la velocidad de corrosión (mm/año) obtenida mediante el método de $\mathrm{R}_{\mathrm{n}}$ con respecto al tiempo de la unión disímil TiC/Cu-Cu y la matriz de cobre en agua de mar sintética con un tiempo de exposición de 24 horas.

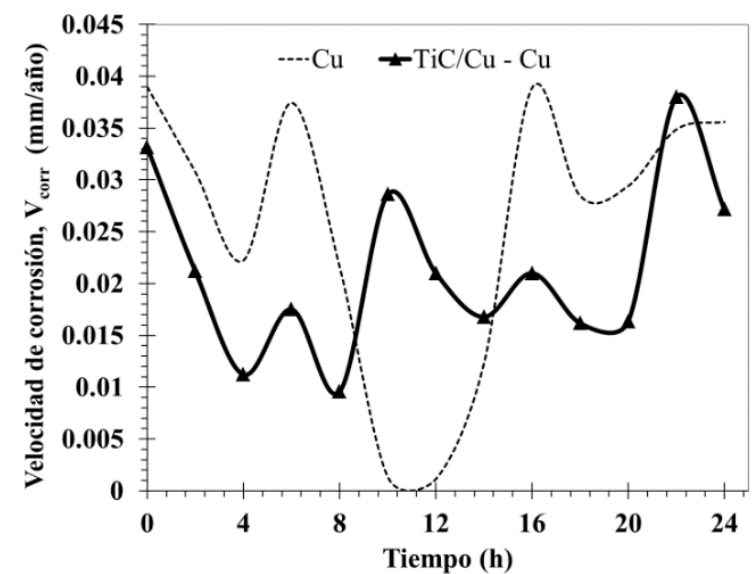

Figura 6: $\mathrm{V}_{\text {corr }}\left(\right.$ obtenida por $\mathrm{R}_{\mathrm{n}}$ ) en función del tiempo de exposición de la unión ( $\mathrm{TiC} / \mathrm{Cu}-\mathrm{Cu}$ ) y cobre.

En la Figura 6 muestra la comparación de la unión y el cobre, se observa un comportamiento similar, esto atribuido a la gran cantidad en área de cobre, el refuerzo de TiC y la presencia de Ag no afectaron considerablemente las propiedades de resistencia a la corrosión del $\mathrm{Cu}$. La unión presenta una variación de $\mathrm{V}_{\text {corr }}$ atribuida a la formación, ruptura y disolución de la película de productos de corrosión y al proceso galvánico generado por entre la plata y el cobre. YU-MEI HAN et al. [15] en sus estudios de corrosión con materiales compuestos, atribuyen este comportamiento la presencia de un material cerámico (interface metal-cerámico) y la presencia de un par galvánico. La disminución de la $\mathrm{V}_{\text {corr }}$ puede ser atribuida a la regeneración de la película de óxidos aunado al empobrecimiento de cobre en la región del compósito.

\subsubsection{Curvas de polarización}

La figura 7 muestra la curva de polarización de la unión $(\mathrm{TiC} / \mathrm{Cu} \bullet \mathrm{Cu})$ inmerso en agua de mar sintética. 


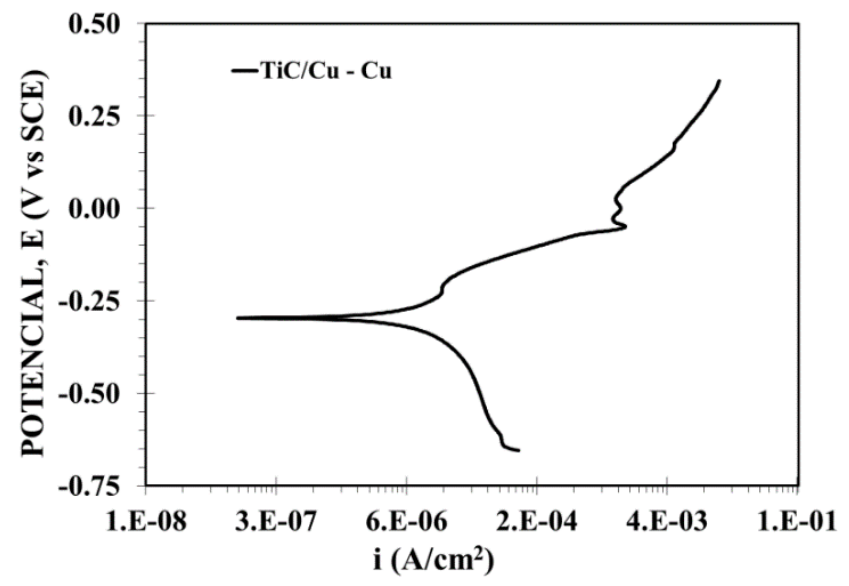

Figura 7: Curvas de polarización de la unión $(\mathrm{TiC} / \mathrm{Cu} \bullet \mathrm{Ag}-\mathrm{Cu} \cdot \mathrm{Cu})$ inmerso en agua de mar sintética durante 24 horas.

Como se observa en la unión brazing composito-cobre se observan tres regiones; 1) la región de disolución activa (en la zona de Tafel), 2) la región de transición activo-pasiva y 3) la región de corriente limite [31]. Conforme aumento el potencial en la región anódica se forma una pasivacion aproximadamente a los $225 \mathrm{mv}$, posteriormente se presenta un proceso de transferencia de carga, a potenciales más altos se presentan dos picos de corriente que pueden atribuirse, el primero, a la formación de $\mathrm{Cu}_{2} \mathrm{O}$ [32], y el segundo, a la formación de oxicloruro de cobre $\left(\mathrm{Cu}_{2}(\mathrm{OH})_{3} \mathrm{Cl}\right)$. En la rama catódica se observa un comportamiento de transferencia de masa, lo cual puede deberse a la difusión del oxígeno sobre la superficie del metal.

\subsubsection{Análisis superficial de la corrosión $\mathrm{TiC} / \mathrm{Cu}-\mathrm{Cu}$ inmerso en agua de mar sintética}

El análisis superficial del proceso de corrosión de las muestras de unión disímil metal-compósito sumergidas en agua de mar sintética mediante microscopia electrónica de barrido, indican que el tipo de corrosión presente es de tres tipos de corrosión localizada.
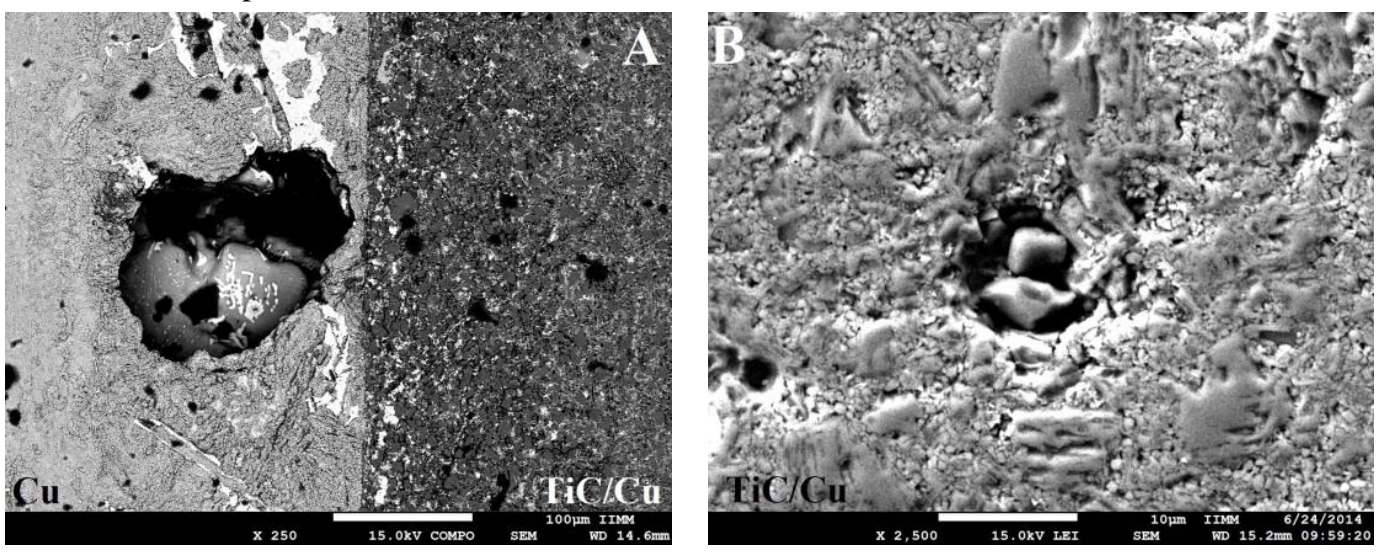

Figura 8: Morfología (SEM) de: Corrosión localizada del tipo picadura del compósito inmerso en agua de mar sintética.

En la Figura 8A se observan dos tipos de corrosión, el primero corrosión por picadura, generada por la formación y ruptura de la película productos de corrosión en la superficie del material, segundo corrosión galvánica generada por acción de la $\mathrm{Ag}$ sobre el $\mathrm{Cu}$ y tercero corrosión por hendidura generada en la interfaz metal-cerámico, como se aprecia en la Figura 8B.

\section{CONCLUSIONES}

Con el objetivo de utilizar un material compuesto en aplicaciones funcionales, se utilizó el compósito TiC/Cu (60-40\% vol.) para producir uniones disimiles por soldadura brazing a partir de $935^{\circ} \mathrm{C}$ y con un tiempo de permanencia de 30 minutos. El arreglo compósito-intercapa-cobre, donde la intercapa es de naturaleza terciaria formada in situ al agregar cantidades mínimas de $\mathrm{Ti}$ en polvo a la laminilla $\mathrm{Ag}-\mathrm{Cu}$ el proceso es por tanto una unión brazing por metal activo. El arreglo, temperatura y tiempo de permanencia permitieron obtener uniones disimiles pero coherentes. 
Con la técnica de Ruido Electroquímico fue posible realizar un seguimiento del proceso corrosivo y mediante el método de resistencia del ruido $(\mathrm{Rn})$, es posible realizar un seguimiento de la velocidad de corrosión de las muestras en estudio. Es importante mencionar que la Vcorr de la unión $\mathrm{TiC} / \mathrm{Cu}-\mathrm{Cu}$ comparada con la matriz cobre presentan una tendencia similar durante las 24 horas de exposicion, presentando un valor de corrosión por debajo de los $0.04 \mathrm{~mm} / \mathrm{año}$.

El tipo de corrosión de acuerdo a las técnicas electroquímicas de RE y CP y al análisis por microscopia electrónica de barrido presente fue corrosión localizada atribuida a tres factores, el primero a la corrosión galvánica generada entre la plata y el cobre, el segundo a la corrosión por hendiduras generado en la región del compósito en la interfaz metal-cerámico y tercero corrosión por picadura generada por la formación y ruptura de la película de productos de corrosión $\left(\mathrm{Cu}_{2} \mathrm{O}\right)$ generada en la superficie de la muestra.

\section{AGRADECIMIENTOS}

Se agradece al Consejo Nacional de Ciencia y Tecnología (CONACYT) por el apoyo brindado y al Laboratorio de Materiales Compuestos del Instituto de Investigación en Metalurgia y Materiales de la Universidad Michoacana de San Nicolás de Hidalgo.

\section{BIBLIOGRAFÍA}

[1] RADJA, I., DJELAD, H., MORALLON, E., et al.,"Characterization and electrochemical properties of conducting nanocomposites synthesized from p-anisidine and aniline with titanium carbide by chemical oxidative method", Synthetic Metals, v. 202, pp. 25-32, February 2015.

[2] CHEN, C.L., LIN, C.H. "Effect of $\mathrm{Y}_{2} \mathrm{O}_{3}$ and TiC Reinforcement Particles on Intermetallic Formation and Hardness of Al6061 Composites via Mechanical Alloying and Sintering”, Metallurgical and materials transactions, v. 46A, pp. 3687-3695, August 2015.

[3] JAFARIAN, H., HABIBI-LIVAR, J., RAZAVI, S.H. "Microstructure evolution and mechanical properties in ultrafine grained $\mathrm{Al} / \mathrm{TiC}$ composite fabricated by accumulative roll bonding", Composites, v. 77B, pp. 84-92, March 2015.

[4] KARANTZALIS, A.E., LEKATOU, A., TSIRKA, K. "Solidification observations and sliding wear behavior of vacuum arc melting processed $\mathrm{Ni}-\mathrm{Al}-\mathrm{TiC}$ composites”, Material Characterization, v. 69, pp. 97107, April 2012.

[5] AKHTARA, F., ASKARIA, S.J., SHAHA, K.A., et al., "Microstructure, mechanical properties, electrical conductivity and wear behavior of high volume TiC reinforced Cu-matrix composites", Material Characterization, v. 60, pp. 327-336, 2009.

[6] CUI, B., HUANG, J.H., XIONG, J.H., "Reaction composite brazing of carbon fiber reinforced SiC composite and TC4 alloy using $\mathrm{Ag}-\mathrm{Cu}-\mathrm{Ti}-(\mathrm{Ti}+\mathrm{C})$ mixed powder”, Materials Science \& Engineering, v. 562A, pp. 203-210, November 2013.

[7] FAN, D., HUANG, J., WANG, Y., et al., "Active brazing of carbon fiber reinforced SiC composite and 304 stainless steel with Ti-Zr-Be”, Materials Science \& Engineering, v. 617, pp. 66-72, August 2014.

[8] ZHANG, L., FENG, J., ZHANG, B., et al., “Ag-Cu-Zn alloy for brazing TiC cermet/steel”,Materials Letters, v. 59, pp. 110-113, 2005.

[9] ZHAO, Y.X., WANG, M.R., CAO, J., et al., "Brazing TC4 alloy to $\mathrm{Si}_{3} \mathrm{~N}_{4}$ ceramic using nano-Si $\mathrm{N}_{4}$ reinforced AgCu composite filler", Materials and Design, v. 76, pp. 40-46, 2015.

[10] SHERIF, E.M, ALMAJID, A.A., LATIF, F.H., et al., "Effects of Graphite on the Corrosion Behavior of Aluminum Graphite Composite in Sodium Chloride Solutions", International Journal of electrochemical science, v. 6, pp. 1085 - 1099, April 2011.

[11] LIU, L., XU, J., LI, Z., "Electrochemical Characterization of $\mathrm{Ti}_{5} \mathrm{Si}_{3} / \mathrm{TiC}$ Nanocomposite coating in $\mathrm{HCl}$ Solution”, International Journal of electrochemical science, v. 8, pp. 5086 - 5101, April 2013.

[12] GALVÁN-MARTÍNEZ, R., CABRERA-DE LA CRUZ, D., CONTRERAS, A., et al., "A novel experimental arrangement for corrosion study of X60 pipeline steel weldments at turbulent flow conditions", The International Journal of Corrosion Processes and Corrosion Control, v. 51, pp. 400-407, May 2016.

[13] HA, H.-Y., KANG, J.-Y., YANG, J., et al., "Limitations in the use of the potentiodynamic polarisation curves to investigate the effect of $\mathrm{Zn}$ on the corrosion behaviour of as-extruded $\mathrm{Mg}-\mathrm{Zn}$ binary alloy", Corrosion Science, v. 75, pp. 426-433, 2013.

[14] TANG, Y., LI, G. "Thermodynamic Study of Sn-Ag-Ti Active Filler Metals”, Physics Procedia, v. 25, 
pp. 30-35, 2012.

[15] HAN, Y.-M., GALLANT, D., CHEN, X.-G.,"Galvanic corrosion associated with Al-B ${ }_{4} \mathrm{C}$ composites/SS304 and $\mathrm{Al}-\mathrm{B}_{4} \mathrm{C}$ composites/AA6061 couples in $\mathrm{NaCl}$ and $\mathrm{H}_{3} \mathrm{BO}_{3}$ solutions", Electrochimica Acta, v. 94, pp.134-142, 2013.

[16] BENEDETI, A.V., SUMODJO, P.T.A., NOBE, K., et al., "Electrochemical studies of copper, copperaluminium and copper-aluminium-silver alloys: impedance results in $0.5 \mathrm{M} \mathrm{NaCl}$ ", Electrochimica Acta, v. 40, pp. 2657-2668, 2005.

[17] BADAWY, W.A., ISMAIL, K.M., FATHI, A.M. "Effect of Ni content on the Corrosion Behaviour of Cu-Ni Alloys in Neutral Sulfate Solutions”, Electrochimica Acta, v. 51, pp. 4182-4189, 2006. 